\title{
Fast Spatial Combinative Lifting Algorithm of the Wavelet Transform with the 9/7 Filter for Image Blocks
}

\section{Hongying Meng and Zhihua Wang}

A new fast spatial Combinative lifting algorithm (SCLA) of the wavelet transform with the 9/7 filter for image blocks is proposed. In comparison with its lifting-based implementation, the multiplication is reduced by a ratio of 5/12. The SCLA can speed up the implementation of the wavelet transform. Introduction: The popular 9/7 filter is one of the biorthogonal wavelet filters proposed by Cohen, Daubechies and Feauveau [1] in 1992. It has been used as the default filter in the irreversible wavelet transform of the upcoming new still image compression standard JPEG2000 [2]. The implementation of the wavelet transform with the 9/7 filter has two modes: a convolution-based [3] and a lifting-based implementation (LBI) [4]. The former has more computation cost than the latter. It is reported that the JPEG2000 encoder takes approximately 34 times longer than the JPEG encoder [2]. The reason is mainly due to the wavelet transform. This letter proposes a new fast spatial combinative lifting algorithm (SCLA) for the wavelet transform with the $9 / 7$ filter in the image blocks compression. In comparison with the LBI, the multiplication is reduced by a ratio of $5 / 12$.

LBI with decomposition level one:

For a 1-D signal $X=\{x(k) \mid k=0,1, \cdots, N-1\}$ with length $N$ (assume $N=2^{L}$ and $L$ is a natural number). If the symmetric extension method on the boundary is chosen, the LBI with decomposition level one by the $9 / 7$ filter can have the following matrix representation:

$Y=T_{N \times N} X=E D C B A X$

where $Y=\{y(k) \mid k=0,1, \cdots, N-1\}$ is the obtained coefficient vector and $T_{N \times N}$ is the associated wavelet transform operator matrix. $A, B, C, D, E$ are the constant matrices associated with each step of the LBI.
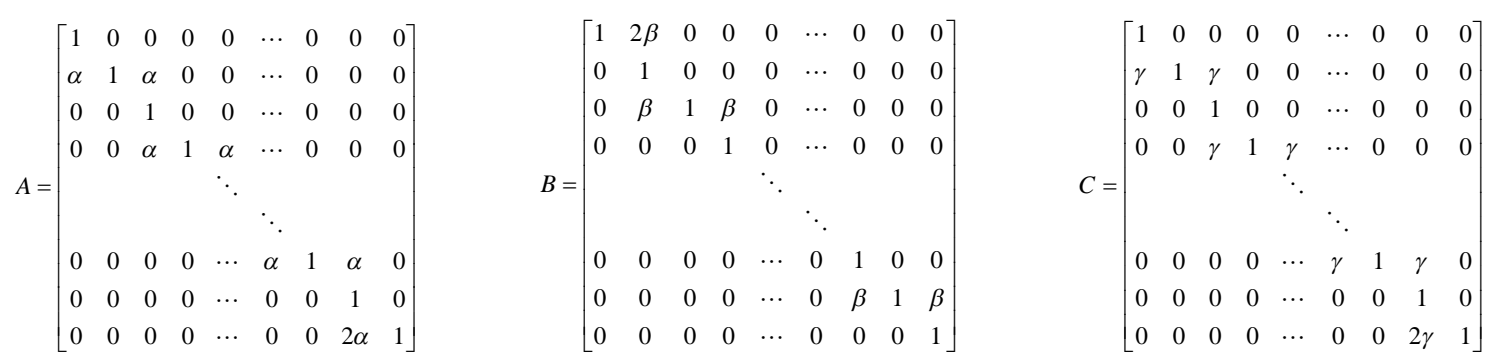


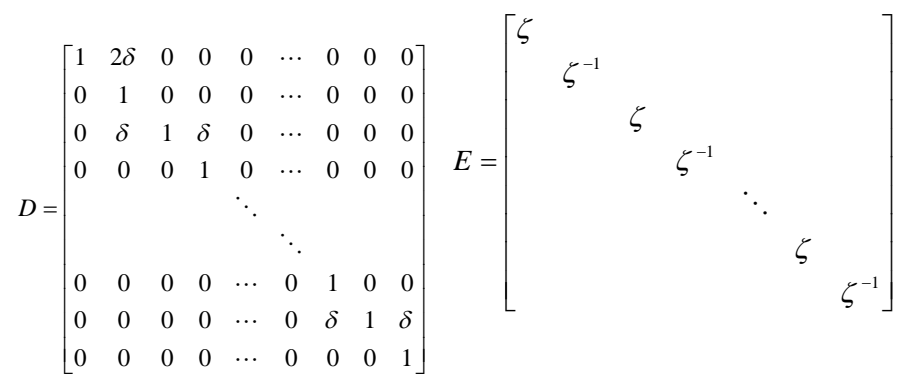

The values of the parameters are:

$$
\left\{\begin{array}{c}
\alpha=-1.586134342 \\
\beta=-0.0529801185 \\
\gamma=0.8829110762 \\
\delta=0.4435068522 \\
\zeta=1.149604398
\end{array}\right.
$$

in which $\zeta$ is a scaling factor. It is worth mentioning that the scaling factor sometimes can be chosen as:

$\zeta^{\prime}=\zeta / \sqrt{2}=0.812893$

For a 2-D image block $M=\{m(i, j) \mid i, j=0,1, \cdots, N-1\}$, the forward wavelet transform of the LBI with decomposition level one by the $9 / 7$ filter can be represented as:

$T_{N \times N} \times M \times T^{t}{ }_{N \times N}=(E D C B A) \times M \times(E D C B A)^{t}$

in which the subscript " $t$ " stands for the transpose of the matrix.

SCLA with decomposition level one:

Utilizing the combinative law of the matrix multiplication, Equation 4 can be represented as:

$T_{N \times N} \times M \times T^{t}{ }_{N \times N}=E\left(D\left(C\left(B\left(A \times M \times A^{t}\right) B^{t}\right) C^{t}\right) D^{t}\right) E^{t}$

The computing process of the SCLA starts from the center and extends to the two sides in Equation 5 . The matrix $A, B, C, D$ and $E$ will be used step by step and their associated obtained image blocks are denoted as $M_{a}, M_{b}, M_{c}, M_{d}$ and $M_{e}$ respectively in the following.

For all $i=0,1, \cdots N / 2-1$ and $j=0,1, \cdots N / 2-1$, the four steps of the SCLA for matrix $A$ are:

$\left\{\begin{array}{l}m_{a}(2 i, 2 j)=m(2 i, 2 j) ; \\ m_{a}(2 i, 2 j+1)=m(2 i, 2 j+1)+\alpha\left[m_{a}(2 i, 2 j)+m_{a}(2 i, 2 j+2)\right] ; \\ m_{a}(2 i+1,2 j+1)=m(2 i+1,2 j+1)+\alpha\left[m_{a}(2 i, 2 j+1)\right. \\ \left.\quad+m_{a}(2 i+2,2 j+1)+m(2 i+1,2 j)+m(2 i+1,2 j+2)\right] \\ m_{a}(2 i+1,2 j)=m(2 i+1,2 j)+\alpha\left[m_{a}(2 i, 2 j)+m_{a}(2 i+2,2 j)\right]\end{array}\right.$

and the four steps for matrix $B$ are: 


$$
\left\{\begin{array}{l}
m_{b}(2 i+1,2 j+1)=m_{a}(2 i+1,2 j+1) \\
m_{b}(2 i+1,2 j)=m_{a}(2 i+1,2 j) \\
\quad+\beta\left[m_{b}(2 i+1,2 j-1)+m_{b}(2 i+1,2 j+1)\right] \\
m_{b}(2 i, 2 j)=m_{a}(2 i, 2 j)+\beta\left[m_{b}(2 i-1,2 j)+m_{b}(2 i+1,2 j)\right. \\
\left.\quad+m_{a}(2 i, 2 j-1)+m_{a}(2 i, 2 j+1)\right] \\
m_{b}(2 i, 2 j+1)=m_{a}(2 i, 2 j+1) \\
\quad+\beta\left[m_{b}(2 i-1,2 j+1)+m_{b}(2 i+1,2 j+1)\right]
\end{array}\right.
$$

Fig.1 can easily show the steps of the SCLA for matrix A and matrix B. The numbers in Fig. 1 are corresponding to the step sequence numbers of the SCLA stated above.

Fig. 1 The step sequence numbers of the SCLA

The steps of the SCLA for matrix $C$ and $D$ are similar with the ones for matrix $A$ and $B$ just by replacing the parameters $\alpha$ with $\gamma$ and $\beta$ with $\delta$.

The steps of the SCLA for matrix $E$ are:

$$
\left\{\begin{array}{c}
m_{e}(2 i+1,2 j)=m_{d}(2 i+1,2 j) ; \\
m_{e}(2 i, 2 j+1)=m_{d}(2 i, 2 j+1) ; \\
m_{e}(2 i, 2 j)=m_{d}(2 i, 2 j) \times \eta ; \\
m_{e}(2 i+1,2 j+1)=m_{d}(2 i+1,2 j+1) / \eta ;
\end{array}\right.
$$

where $\eta=\zeta^{2}$.

Up to now, the SCLA of the wavelet transform with decomposition level one for the 2-D image $M$ is completed. Rearranged the order of the coefficients in matrix $M_{e}$, the coefficients matrix can be divided into four sub-bands denoted as $L L, H L, L H$ and $H H$. With the decomposition level increases one, the SCLA will be used in the same manner for the $L L$ sub-band of the matrix.

The inverse transform of the SCLA can immediately be derived from the forward transform by running each steps of the SCLA backward just as the one of the LBI. The integer version of the SCLA also can be derived by rounding at each step just as the one of the LBI. They both are omitted here.

\section{The number of multiplication analysis:}

If the decomposition level is one, it can be easily calculated that the number of the multiplication by the LBI for 2-D image block $M$ is $6^{*} N^{*} N$. In the SCLA, the number for each matrix $A, B, C$ and $D$ is $3 / 4^{*} N^{*} N$. The number for matrix $E$ is $1 / 2^{*} N^{*} N$. So the number of multiplication by the SCLA is $3.5 N^{*} N$.

For the decomposition level $J(J \leq L)$, the total multiplication by the LBI and by the SCLA are $2^{*}\left(4-4^{-J+1}\right)^{*} N^{*} N$ and $4 / 3^{*}\left(4-4^{-J+1}\right)^{*} N^{*} N$ respectively. The ratio of the SCLA to LBI is still $7 / 12$. The 
comparison is listed in Table 1.

Table 1: The multiplication comparison of the $L B I$ and SCLA

Hongying Meng and Zhihua Wang (Department of Electronic Engineering, Tsinghua University, Beijing, 100084, People's Republic of China)

E-mail: menghy@ee.tsinghua.edu.cn

\section{Preference}

1 COHEN A., DAUBECHIES I. and FEAUVEAU J., "Biorthogonal bases of compactly supported wavelets", Comm. Pure Appl. Math., 1992, 45, pp.485-560

2 SKODRAS A. N., CHRISTOPOULOS C. A. and Ebrahimi T., "JPEG2000: the up coming still image compression standard", Proceedings of the 11th portuguese conference on pattern recognition (RECPA00D 20), Porto, Portugal, 11 May 2000, pp.359-366

3 MALLAT S., "A theory for multiresolution signal decomposition: the wavelet representation", IEEE Trans. on Pattern Analysis and Machine Intelligence, 1989, 11, pp.674-693

4 DAUBECHIES I. and SWELDENS W., "Factoring wavelet transforms into lifting steps", J. Fourier Anal. Appl., 1998, 4, (3), pp.247-269

\begin{tabular}{|l|l|l|l|l|l|l|l|}
\hline 1 & 2 & 1 & 2 & 1 & 2 & 1 & 2 \\
\hline 4 & 3 & 4 & 3 & 4 & 3 & 4 & 3 \\
\hline 1 & 2 & 1 & 2 & 1 & 2 & 1 & 2 \\
\hline 4 & 3 & 4 & 3 & 4 & 3 & 4 & 3 \\
\hline 1 & 2 & 1 & 2 & 1 & 2 & 1 & 2 \\
\hline 4 & 3 & 4 & 3 & 4 & 3 & 4 & 3 \\
\hline 1 & 2 & 1 & 2 & 1 & 2 & 1 & 2 \\
\hline 4 & 3 & 4 & 3 & 4 & 3 & 4 & 3 \\
\hline
\end{tabular}

(a) for matrix $A$ and $C$

\begin{tabular}{|l|l|l|l|l|l|l|l|}
\hline 3 & 4 & 3 & 4 & 3 & 4 & 3 & 4 \\
\hline 2 & 1 & 2 & 1 & 2 & 1 & 2 & 1 \\
\hline 3 & 4 & 3 & 4 & 3 & 4 & 3 & 4 \\
\hline 2 & 1 & 2 & 1 & 2 & 1 & 2 & 1 \\
\hline 3 & 4 & 3 & 4 & 3 & 4 & 3 & 4 \\
\hline 2 & 1 & 2 & 1 & 2 & 1 & 2 & 1 \\
\hline 3 & 4 & 3 & 4 & 3 & 4 & 3 & 4 \\
\hline 2 & 1 & 2 & 1 & 2 & 1 & 2 & 1 \\
\hline
\end{tabular}

(b) For matrix $B$ and $D$

Fig. 1 The step sequence numbers of the SCLA

Table 1: The multiplication comparison of the $L B I$ and SCLA

\begin{tabular}{|c|c|c|}
\hline Algorithm & Level $=1$ & Level $=J$ \\
\hline LBI & $\left(N / 2^{*} 4+N\right)^{*} 2 N=6 N^{*} N$ & $2^{*}\left(4-4^{-J+1}\right)^{*} N^{*} N$ \\
\hline SCLA & $3 N^{*} N+N^{*} N / 2=3.5 N^{*} N$ & $7 / 6^{*}\left(4-4^{-J+1}\right)^{*} N^{*} N$ \\
\hline
\end{tabular}

